\title{
Calidad de Cardo Mariano (Silybum marianum (L.) Gaertn.) Cosechado en Diferentes Estados Fenológicos
}

\author{
Felícitas Hevia $^{(1)}$, Rosemarie L. Wilckens ${ }^{(2)}$, Marisol T. Berti ${ }^{(2)}$ y Susana U. Fischer ${ }^{(2)}$ \\ Universidad de Concepción, (1) Fac. de Ingeniería Agrícola, (2) Fac. de Agronomía, Casilla 537, \\ Chillán-Chile (e-mail: fhevia@udec.cl)
}

\begin{abstract}
Resumen
Se cultivaron dos genotipos de cardo mariano (Silybum marianum (L.) Gaertn.), alemán y chileno, con el propósito de determinar el momento más adecuado de cosecha. Se usó un diseño de parcela dividida con bloques aleatorios en un arreglo factorial de $4 \times 2$ y cuatro repeticiones. La parcela principal correspondió al momento de cosecha (50\% de plantas con 2, 4, 6 y 10 capítulos maduros) y la sub-parcela al genotipo (alemán y chileno). A la cosecha se evaluó el peso de 1000 semillas, el porcentaje de semillas maduras y el contenido de silimarina. Siempre el genotipo alemán presentó una mayor proporción de semillas maduras, las que fueron más pesadas que las del genotipo chileno. Sin embargo, en este último el contenido de silimarina fue superior. Se concluyó que el momento más adecuado para cosechar ambos genotipos fue el estado de cuatro capítulos maduros.
\end{abstract}

\section{Quality of Milk Thistle (Silybum marianum (L.) Gaertn.) Harvested in Different Phenological Stages}

\begin{abstract}
Two milk thistle genotypes (Silybum marianum (L.) Gaertn.), German and Chilean, were cultivated to determine the most suitable moment of harvest. A randomized complete block design with a split plot arrangement and four replicates was used. The main plot was harvested timeliness (2, 4, 6 y 10 mature capitula in $50 \%$ of the plants) and the subplot was the genotype. For the harvest, the weight of 1000 seeds, the percentage of mature seeds and the silymarin content were evaluated. Always the German genotype showed higher mature seed proportion and with higher weight than the Chilean one. However, the Chilean genotype seeds showed higher silymarin content. This study concluded that the most adequate moment for harvest is the physiological state of four mature capitula for both genotypes.
\end{abstract}

Keywords: milk thistle, silymarin, phenological stages, harvest time 


\section{INTRODUCCIÓN}

De los frutos del cardo mariano (Silybum marianum L. Gaertn.) se extrae un principio activo, conocido con el nombre de silimarina, el cual se ha usado durante miles de años para tratar los problemas del hígado. Reconocidas son sus propiedades antihepatotóxica, antiinflamatoria, antioxidante, antitumoral y hepatoprotectoras. Promueve el crecimiento de nuevas células del hígado, por lo que se recomienda en el tratamiento de hepatitis, cirrosis y cuando se ingieren drogas que pueden causar daño hepático como efecto colateral (Blumenthal et al., 2000; Carrier et al., 2002; Curioni et al., 2002; Kvasnicka et al., 2003; Tong-Chun et al., 2006).

La silimarina comprende a cuatro flavolignanos con estructura química similar: silibina, isosilibina, silicristina y silidianina, siendo prácticamente el $50 \%$ de la mezcla silibina, que es el compuesto con mayor actividad farmaceútica (Kvasnicka et al., 2003). El contenido de silimarina en los aquenios fluctúa entre 1,5 y 3,0\% y depende de factores genéticos, la disponibilidad de agua en el suelo y del estado de madurez al momento de la cosecha (Spitzová y Starý, 1985; Chiavari et al., 1991; Hammouda et al., 1991; Tong-Chun et al., 2006).

El desarrollo y la maduración de los capítulos no ocurren en forma simultánea. Primero madura el capítulo del tallo principal, mientras en los estratos inferiores, algunos capítulos aún están cerrados. El fácil desprendimiento de los frutos desde los capítulos maduros puede causar una pérdida importante de la cosecha (Dodd, 1989; Curioni y Arizio, 2000; Curioni et al., 2002).

El objetivo de esta investigación fue evaluar el peso de 1000 semillas, porcentaje de semillas maduras y contenido de silimarina en dos genotipos (alemán y chileno) de cardo mariano, cosechados en diferentes estados fenológicos.

\section{MATERIALES Y MÉTODOS}

\section{Manejo del cultivo}

El ensayo se realizó en la Estación Experimental de la Facultad de Agronomía, Campus Chillán, de la Universidad de Concepción (144 m.s.n.m., 36 $34^{\circ} \mathrm{S}$ y $72^{\circ} 06^{\prime} \mathrm{W}$ ); temperatura media anual $13,9^{\circ} \mathrm{C}$; temperatura máxima (enero) $29-32^{\circ} \mathrm{C}$; temperatura mínima (julio) $3,8^{\circ} \mathrm{C}$; precipitación anual $900-1100$ $\mathrm{mm}$; en un suelo de la Serie Arrayán (Typic Haploxerand) con topografía plana, buen drenaje; $\mathrm{N}-\mathrm{NO}_{3}$, $6,5 \mathrm{mg} \mathrm{kg}{ }^{-1}$; P Olsen, 29,7 mg kg${ }^{-1}$; y K, 0,61 $\mathrm{cmol} \mathrm{kg}^{-1}$. Se sembró el 28 de abril del 2000, un genotipo de origen alemán y otro chileno (silvestre, recolectado en la misma localidad) a $60 \mathrm{~cm}$ entre hileras y $20 \mathrm{~cm}$ sobre la hilera, en dosis equivalente a 84000 semillas ha $^{-1}$. Se aplicó al voleo urea al estado de dos hojas verdaderas $\left(50 \mathrm{~kg} \mathrm{~N} \mathrm{ha}^{-1}\right)$ y al inicio de la emisión del tallo floral (100 $\left.\mathrm{kg} \mathrm{N} \mathrm{ha}^{-1}\right)$. Se controló la maleza en forma manual, se regó cada 7 días por tendido entre el 28 de octubre y el 9 de diciembre del 2000, no se controló plagas.

\section{Diseño experimental}

Parcela dividida con bloques aleatorios en arreglo factorial $4 \times 2$. La parcela principal correspondió al momento de cosecha (50\% de las plantas con 2, 4, 6 y 10 capítulos maduros) y la subparcela al genotipo (alemán y chileno) y cuatro repeticiones. Cada subparcela constó de cuatro hileras de $5 \mathrm{~m}$ de largo. Entre repeticiones se dejaron libre franjas de $3 \mathrm{~m}$. Manualmente se cosechó $3 \mathrm{~m}$ lineales de las hileras centrales.

Peso de mil semillas

Se contaron al azar mil semillas de cada repetición y se pesaron en una balanza analítca con sensibilidad 0,001 $\mathrm{g}$.

\section{Porcentaje de semilla madura}

En $50 \mathrm{~g}$ de muestra se separaron manualmente las semillas maduras (color marrón) y se pesaron.

\section{Análisis de silimarina}

Se determinó mediante un método descrito por Chiavari et al. (1991), se informó como \% de silibina. 


\section{Análisis estadístico}

Los datos se sometieron a un análisis de varianza y cuando correspondía se aplicó el test DMS (Diferencias mínimas significativas) para la comparación de medias.

\section{RESULTADOS Y DISCUSIÓN}

Se observó algunas diferencias entre las características agronómicas de ambos genotipos. El alemán fue más precoz y se cosechó siete días antes; siempre presentó un tallo floral más alto (168 versus $137 \mathrm{~cm}$ ); desarrolló menos capítulos (13 versus 22); mientras que la pérdida de semilla fue menor (30,3 versus $42,3 \%$ ), aumentando en ambos genotipos a medida que se atrasó la cosecha. Los mayores rendimientos se observaron en las dos primeras cosechas, $1680 \mathrm{~kg} \mathrm{ha}^{-1}$ el alemán y 1887 $\mathrm{kg} \mathrm{ha}^{-1}$ el chileno. Se observó una alta variabilidad en los parámetros estudiados, particularmente, en el genotipo chileno lo que se puede atribuir al hecho que las plantas se obtuvieron de semilla colectada de plantas silvestres.

Peso de mil semillas

El análisis de varianza arrojó diferencias $(P \leq 0,05)$ para momento de cosecha, genotipo e interacción entre ambos factores. El peso de mil semillas promedio del genotipo alemán superó al del chileno, 24,0 y 20,2 g, respectivamente (Tabla 1). En el genotipo alemán no se observó diferencia $(P>0,05)$ entre $M_{1}$ y $M_{2}$, sin embargo, ambos fueron más pesadas que $M_{3}$ y $M_{4}$. En cambio, el genotipo chileno no presentó deferencias entre los momentos de cosecha. Se ha informado que el peso de mil semillas de cardo mariano varía entre 13,5 y 31,0 g, encontrándose ambos genotipos en este rango (Spitzová y Starý, 1985; Dachler y Pelzmann, 1989; Chiavari et al., 1991; Curioni y Arizio, 2000; Carrubba et al. ,2002).

En general, el peso y tamaño final de cualquier fruto depende de la posición relativa de las flores, el tamaño del ovario antes de la antesis, el orden de polinización y la competencia entre frutos por los fotoasimilados (Azcon-Bieto y Talon, 1993). Así, el tamaño y peso de mil semillas en capítulos de maravilla disminuye desde el exterior al interior del disco, habiendo en el centro un mayor porcentaje de semillas estériles, estableciéndose diferencias en el peso y tamaño de las semillas de un mismo capítulo y entre capítulos de distinto tamaño (Connor y Hall, 1997; Seiler, 1997).

\section{Porcentaje de semilla madura}

El análisis de varianza detectó diferencias $(P \leq 0,05)$ para el momento de cosecha e interacción genotipo-momento de cosecha.

De la Tabla 1 se desprende que a la primera cosecha $\left(\mathrm{M}_{1}\right)$ el genotipo chileno presentó más semillas maduras (42,4\%) que el alemán (24,5\%). En cambio, en $M_{2}$ y $M_{3}$ se invirtió esta situación, presentando mayor cantidad de semillas maduras el genotipo alemán. Sin embargo, en $M_{4}$ no se observó diferencia entre los genotipos.

El genotipo alemán alcanzó los más altos porcentajes de semilla madura en $M_{3}$ y $M_{4}(75,7$ y $87,2 \%$, respectivamente) y el chileno en $M_{4}(84,2 \%)$, sin diferenciarse en este último $M_{1}, M_{2}$ y $M_{3}$, que fluctuaron entre 42,4 y $53,2 \%$.

El tiempo transcurrido desde postantesis a maduración en ambos genotipos fue de 17 días. Sin embargo, en el genotipo alemán se observó una proporción mayor de semilla madura en el mismo estado de desarrollo a medida que se postergó la cosecha (Tabla 1). Esto indica que la senescencia de la planta se inició antes en el genotipo alemán. En forma paralela se sintetizaron los pigmentos que colorean al fruto (Azcon-Bieto y Talon, 1993).

Contenido de silimarina

El contenido de silimarina, expresado como \% silibina, presentó una alta variabilidad entre las repeticiones de los distintos tratamientos. Por esta razón, aún cuando a veces ocurrieron variaciones 
importantes en la concentración del metabolito, éstas no fueron significativas. La variabilidad observada se atribuyó a la madurez no uniforme de la semilla para un mismo momento de cosecha y

a la ausencia de mejoramiento genético (genotipo chileno) o mejoramiento orientado hacia rendimiento de semilla (genotipo alemán).

Tabla 1: Peso de mil semillas y porcentaje de semilla madura en cardo mariano de los genotipos

alemán y chileno, en relación con el momento de cosecha. *Letras mayúsculas distintas en sentido

horizontal indican diferencia (DMS, $\mathrm{P} \leq 0,05)$; ** Letras minúsculas distintas en sentido vertical indican

diferencia entre sí (DMS, $P \leq 0,05)$.

\begin{tabular}{|c|c|c|c|c|}
\hline \multirow{2}{*}{ Tratamiento } & \multirow{2}{*}{$\begin{array}{c}\text { Momento cosecha } \\
\text { No capítulos maduros }\end{array}$} & \multicolumn{2}{|c|}{ Genotipo } & \multirow{2}{*}{$\begin{array}{c}\text { Promedio } \\
\text { Momento cosecha }\end{array}$} \\
\hline & & Alemán & Chileno & \\
\hline \multicolumn{5}{|c|}{ Peso mil semillas (g) } \\
\hline $\mathrm{M}_{1}$ & 2 & $25,9 A^{\star} a^{\star \star}$ & $20,8 \mathrm{Ba}$ & 23,4 \\
\hline $\mathrm{M}_{2}$ & 4 & $25,4 \mathrm{Aa}$ & $19,5 \mathrm{Ba}$ & 22,4 \\
\hline$M_{3}$ & 6 & $23,0 \mathrm{Ab}$ & $20,1 \mathrm{Ba}$ & 21,5 \\
\hline $\mathrm{M}_{4}$ & 10 & $21,9 \mathrm{Ab}$ & $20,5 \mathrm{Aa}$ & 21,2 \\
\hline Promedio & & 24,0 & 20,2 & 22,1 \\
\hline \multicolumn{5}{|c|}{ Porcentaje de semilla madura (\%) } \\
\hline$M_{1}$ & 2 & $24,5 \mathrm{Bc}$ & $42,4 \mathrm{Ab}$ & 33,4 \\
\hline $\mathrm{M}_{2}$ & 4 & $56,1 \mathrm{Ab}$ & $39,4 \mathrm{Bb}$ & 47,7 \\
\hline $\mathrm{M}_{3}$ & 6 & $75,7 \mathrm{Aa}$ & $53,2 \mathrm{Bb}$ & 64,5 \\
\hline $\mathrm{M}_{4}$ & 10 & $87,2 \mathrm{Aa}$ & $84,2 \mathrm{Aa}$ & 85,7 \\
\hline Promedio & & 60,9 & 54,8 & 57,8 \\
\hline
\end{tabular}

Tabla 2: Contenido de silimarina (\% de silibina) en semilla de cardo mariano de los genotipos alemán y chileno, en relación con el número de capítulos maduros al momento de cosecha. *Letras distintas en sentido horizontal indican diferencias según Test DMS, con $\mathrm{P} \leq 0,05$.

\begin{tabular}{ccccc}
\hline \multirow{2}{*}{ Tratamiento } & Momento cosecha & \multicolumn{2}{c}{ Genotipo } & \multirow{2}{*}{ Promedio } \\
\cline { 3 - 3 } & No capítulos maduros & Alemán & Chileno & \\
\hline & & & & \\
$M_{1}$ & 2 & 4,41 & 5,07 & 4,74 \\
$M_{2}$ & 4 & 4,95 & 5,03 & 4,99 \\
$M_{3}$ & 6 & 4,44 & 4,93 & 4,69 \\
$M_{4}$ & 10 & 4,10 & 5,24 & 4,67 \\
Promedio & & $4,48 \mathrm{a}^{*}$ & $5,07 \mathrm{~b}$ & \\
\hline
\end{tabular}

El análisis de varianza indicó diferencias entre los genotipos, no hubo interacción entre los factores estudiados. El contenido de silimarina promedio de las cuatro cosechas, expresado como \% de silibina, fue superior en el genotipo chileno, fluctuó entre 4,93 y 5,24 5 (Tabla 2). 
En el genotipo alemán varió entre 3,7 y 5,7\%, con un valor promedio igual a 4,5\%, mientras que en el genotipo chileno fluctuó entre 4,3 y $5,7 \%$, con un promedio de $5,1 \%$.

No se observó diferencia entre momento de cosecha en el contenido promedio de ambos genotipos. Estos valores fueron más altos que los informados por Chiavari et al. (1991) y Carrier et al. (2002), que fluctúan entre 2,6 y $4,3 \%$ de silibina para semilla de cardo mariano de distinta procedencia cultivado en Italia. El menor contenido de silimarina del genotipo alemán se podría explicar por el mayor peso de la semilla, lo cual habría provocado un efecto de dilución del metabolito secundario. El peso de silimarina $(\mathrm{mg})$ por semilla fue superior en el genotipo alemán, excepto en la última cosecha. Spitzová y Starý (1985), indicaron que se observa una mayor concentración de silimarina

cuando el peso de 1000 semillas es superior a 25,1 g. La semilla del genotipo alemán superó este valor en las dos primeras cosechas, alcanzando 4,95\% en $\mathrm{M}_{2}$.

En cambio, el peso de mil semillas del genotipo chileno siempre estuvo bajo ese valor y el contenido de silimarina entre 4,93-5,24 \%. Cabe destacar, que la mayor concentración de silimarina se observó cuando el peso de mil semillas fue 20,5 g. La menor concentración de silimarina del genotipo alemán podría obedecer al hecho que los esfuerzos del mejoramiento estuvieron orientados hacia un mejor rendimiento de semilla, lo que habría afectado negativamente la calidad de la misma (Gabucci et al., 2002).

\section{CONCLUSIONES}

Bajo las condiciones en que se realizó el estudio, el momento más apropiado para cosechar ambos genotipos fue el estado de cuatro capítulos maduros $\left(\mathrm{M}_{2}\right)$, cuando se alcanzó el $5 \%$ de silibina.

Es posible atrasar la cosecha del genotipo chileno hasta el estado de seis capítulos maduros $\left(M_{3}\right)$, sin desmedro de la calidad y rendimiento de semilla.

\section{REFERENCIAS}

Azcon-Bieto, J. y M. Talon; Fisiología y Bioquímica Vegetal. McGraw-Hill. Madrid, España (1993).

Blumenthal, M., A. Goldberg y J. Brinckmann; Milk thistle fruit. In: Herbal Medicine. American Botanical Council. Austin, 257-263, Texas-USA (2000).

Carrier, D.; T. Crowe; S. Sokhansanj; J. Wahab y B. Barl; Milk thistle, Sylibum marianum (L.) Gaertn., flower head development and associated marker compound profile. J. Herbs, Spices \& Medicinal Plants 10 (1) 65-74 (2002).

Carrubba, A. R. La Torre y A. Matranga; Cultivation trials of some aromatic and medicinal plants in semiarid Mediterranean environment. Acta Horticulturae 576: 207-213 (2002).

Chiavari, G., G.C. Galletti, M. Marotti y R. Piccaglia; Silymarin content of different Silybum marianum (L.) Gaertn. cultivars. Herba Hungarica 30(1-2), 23-34 (1991).

Connor, D.J. y A.J. Hall; Sunflower Physioloy. In: A. Schneiter (Ed.). Sunflower Technology and Production. American Society of Agronomy. p. 113-115, Monograph No 35 (1997).

Curioni, A. y O. Arizio; Producción argentina de cardo mariano. Panorama Agrario Mundial 24 (211),42-48 (2000). http://www.inta.gov.ar/Econ/ pam/pam211_res.htm\#cardo. (Acceso 22 de agosto (2001). 
Curioni, A. M. García, W. Alfonso y O. Arizio; Predicción de la cosecha de cardo mariano a través de las características externas que presentan los capítulos. Acta Horticulturae 569:257-261 (2002).

Dachler, M. y H. Pelzmann; Heil und Gewürzpflanzen: Anbau, Ernte Aufbereitung. Österreichischer Agrarverlag. Austria (1989).

Dodd, J.; Phenology and seed production of variegated thistle, Silybum marianum (L.) Gaertn., in Australia in relation to mechanical and biological control. Weed Res. 29, 255-263 (1989).

Gabucci, L, A. Curioni, M. García y M.E. Urrutia; Producción de semillas en el cultivo de cardo mariano. Acta Horticulturae 569, 121-128 (2002).

Hammouda, F.M. y otros 5 autores; Evaluation of the silymarin content in Silybum marianum cultivated under different agricultural conditions. Planta Med. 57(2), A29 (1991).

Kvasnicka, F. y otros 4 autores; Analysis of the active components of silymarin. J. Chromatogr. A 990, 239-245 (2003).

Seiler, G. J.; Anatomy and Morphology of Sunflower. In: A Schneiter (Ed.) Sunflower Technology and Production. American Society of Agronomy. Monograph № 35, 67-70 (1997).

Spitzová, I. y F. Starý; The content and localization of flavonolignanes in Silybum marianum (L.) Gaertn. in ontogeneseis. Sborník ÚVTIZ-Zahradnictvi 12(4), 301-307 (1985).

Tong-Chun B. y otros 4 autores; Solubility of silybin in aqueous poly(ethylene glycol) solution. International J. Pharmeceutics, 308 (1,2,3), 100-106 (2006). 